\title{
Un ciclo de mejora docente aplicado al estudio del hecho imponible en el Impuesto sobre la Renta de las Personas Físicas
}

\author{
A cycle of educational \\ improvement applied to the \\ study of the taxable event in \\ the Personal Income Tax
}

MARía TERESA MORIES JiMÉNEZ

ORCID: https://orcid.org/0000-0003-1699-6597

Departamento de Derecho Financiero y

Tributario. Universidad de Sevilla.

mories1@us.es

Fecha de recepción:

Fecha de aceptación:

DOI: http://dx.doi.org/10.12795/9788447221912.063

Pp.: 1452-1473 


\section{Resumen}

El Ciclo de Mejora en el Aula (CIMA) presentado en este capítulo aborda el estudio del hecho imponible en el Impuesto sobre la Renta de las Personas Físicas (en adelante IRPF) en la asignatura Impuestos Directos I del Máster de Asesoría jurídico-mercantil, fiscal y laboral (en adelante MAJMFL). A través del planteamiento de un problema central, se desencadenan tres subpreguntas que abordarán los aspectos objetivo, subjetivo y temporal del hecho imponible permitiendo mediante una cuidada planificación de secuencias de actividades de contraste y teniendo en cuenta las ideas de los estudiantes determinantes de la evolución de sus aprendizajes y sus obstáculos, que el alumno conozca los distintos componentes de la renta gravada en el IRPF.

Palabras clave: Derecho Financiero y Tributario; Máster en Asesoría Jurídico-mercantil, fiscal y laboral; docencia universitaria; experimentación docente universitaria; renta gravada.

\section{Abstract}

The Classroom Improvement Cycle (CIMA) presented in this chapter deals with the study of the taxable event in Personal Income Tax. in the subject Direct Taxes I of the Master's Degree in Juridical, Legal, Mercantile and Labour Consultancy. By means of a central problem, three sub-questions are triggered that will address the objective, subjective and temporal aspects of the taxable event, allowing through a careful planning of contrasting activities and taking into account the ideas of the students who determine the evolution of their learning and their obstacles, that the student knows the different components of the income taxed in the Personal Income Tax.

Keywords: Tax Law; Master's Degree in Juridical, Legal, Mercantile and Labour Consultancy; University teaching; University teaching experimentation; Taxed Income. 


\section{Introducción.}

En el presente trabajo se aborda el diseño, desarrollo y evaluación de un CIMA en la asignatura "Impuestos Directos I", del Máster en Asesoría jurídico-mercantil, fiscal y laboral (en adelante MAJMFL), impartido en el primer cuatrimestre del curso 2019/2020. Es una asignatura de 4,5 créditos obligatorios que pretende enriquecer la formación del alumno en una materia que sólo ha podido conocer a un nivel elemental o fundamental en las enseñanzas de Grado. El grupo de alumnos no es excesivamente numeroso (30) lo que permite que se puedan llevar a cabo fácilmente actividades en grupos de trabajo, que unido a que se trata de alumnos que han finalizado sus estudios de grado, consideramos que es un marco en el que la realización del CIMA puede servir para alcanzar unos resultados muy positivos tanto para los alumnos como para el profesor. El ciclo abarcará un total de 8 horas, es decir, 3 de las 9 sesiones que tiene el desarrollo de esta disciplina. En concreto se aplicará en las 3 primeras sesiones, pues por motivos de organización de la docencia así se ha creído más oportuno, haciendo coincidir el comienzo de nuestra asignatura con la aplicación del ciclo. Nuestra intención es, si los resultados que se obtiene resultan especialmente alentadores, plantear las sesiones posteriores a las de este CIMA en el mismo sentido, para completar el desarrollo completo de nuestra disciplina utilizando estas dinámicas de trabajo a las que nos referiremos en las páginas siguientes. 


\section{Diseño previo del CIMA}

\section{Mapa de contenidos}

Este es nuestro mapa de contenidos para el desarrollo del CIMA. La pregunta sobre la que gira es: ¿cuáles son los aspectos fundamentales de la renta gravada por el IRPF?, ¿resuelve el problema de la sujeción al impuesto abarcando los tres aspectos fundamentales del hecho imponible: aspecto material, personal y temporal?

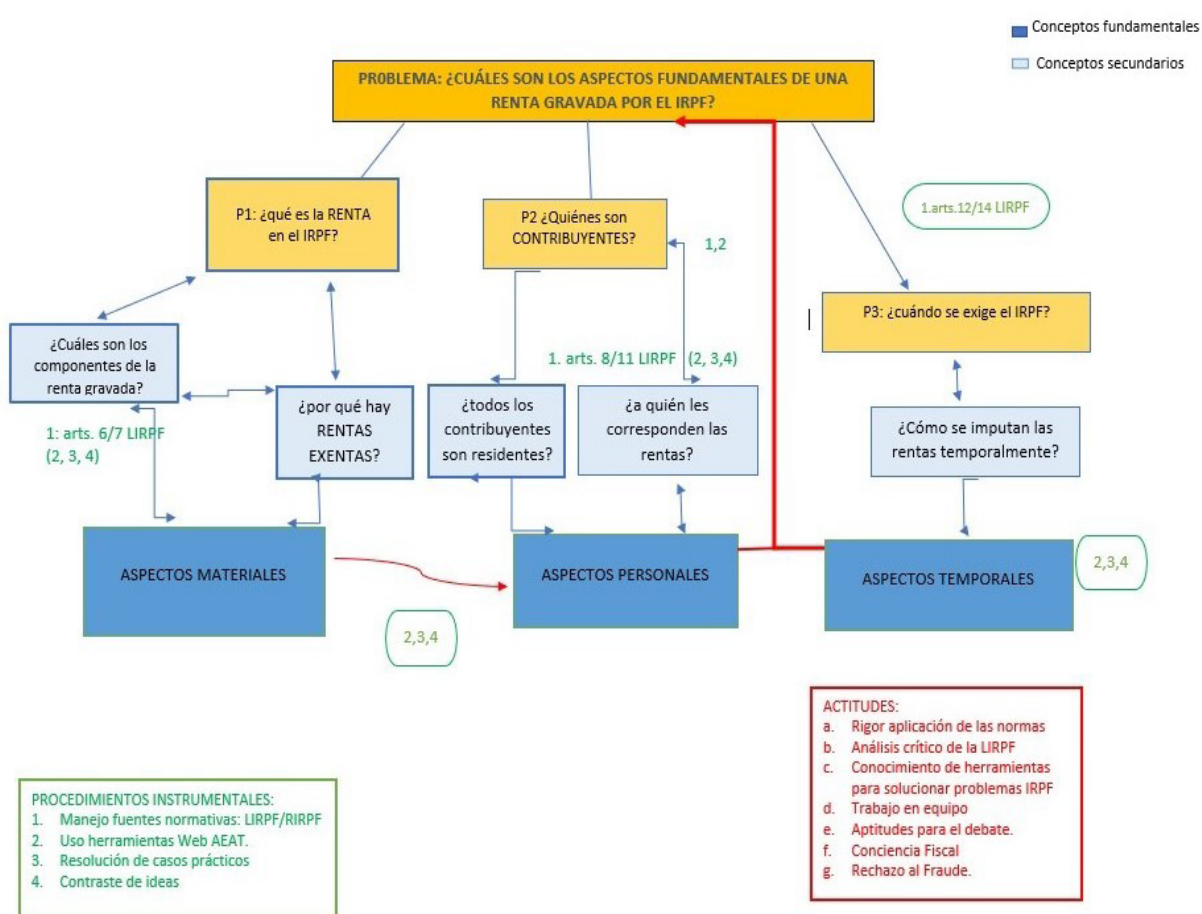

Figura 1. Mapa de contenidos

Aunque hemos tratado de reflejar en algunos puntos del mapa el desarrollo de los procedimientos instrumentales, hemos de señalar que el manejo de fuentes normativas (1) y el uso de herramientas Web AEAT (2) estará 
siempre presente en cualquiera de las actividades que desarrollemos. En cuanto a las actitudes que queremos potenciar, consideramos que se van a ir desarrollando en el marco del CIMA de forma progresiva, siendo las dos últimas reflejadas: conciencia fiscal y rechazo al fraude, las que se consiguen cuando el alumno entiende el significado de la imposición personal sobre la renta.

\section{Modelo metodológico posible para este CIMA}

Tras el desarrollo del primer ciclo y analizar distintos modelos metodológicos en las sesiones llevadas a cabo a lo largo del Curso General de Docencia Universitaria (CGDU), he llegado a la conclusión de que deben ser los alumnos los que tengan el protagonismo principal en las distintas actividades que se programen de manera que partiendo de una serie de preguntas (PR) que formularé inicialmente, en torno a las rentas sujetas al IRPF, seamos capaces de motivar a los alumnos para que sean ellos lo que asumiendo un rol principal participen activamente en el aprendizaje (IA) y adquieran unos conocimientos no solo teóricos, sino instrumentales y actitudinales mucho más amplios y profundos, para que se mantengan en la memoria experiencial y no solo en la memoria académica.

Para ello utilizaremos fundamentalmente textos legales, jurisprudencia y resoluciones de órganos jurisdiccionales y administrativos relevantes en nuestra materia, aplicando las herramientas de la web AEAT, que nos permitan resolver distintos casos prácticos, que hagan avanzar a los alumnos y obtener resultados que resuelvan las cuestiones inicialmente planteadas. Para ello desarrollaremos actividades de contraste $(\mathrm{AC})$ con la participación principal de alumnos y profesor, dejando de lado la posición de "narrador omnisciente" y asumiendo una posición más cercana alumno, pero llevando las riendas de la clase en todo momento e intentando estar en muchas ocasiones en un segundo plano (FINKEL 2008). 
Para finalizar nuestro ciclo, se proponen unas actividades de sintesis para recoger las ideas fundamentales alcanzadas por "todos" en torno a las preguntas y problemas trabajados. Al ser un modelo metodológico circular me permitirá tratar, repitiendo el mismo modelo en cada sesión, los diferentes problemas que he planteado en el mapa de contenidos, lo que puede cambiar serán los tiempos según las actividades que se vayan planteando en cada una de las sesiones.

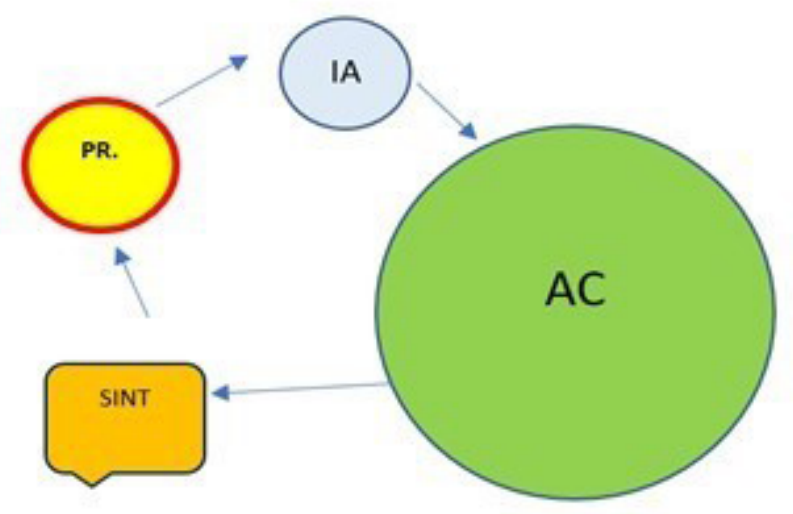

Figura 2: Modelo metodológico

PR: Pregunta que encierra algún/algunos problemas. Se trata de la pregunta inicial que planteada a los alumnos hace que estos empiecen a buscar las posibles soluciones. IA: Ideas de los alumnos. En esta fase del modelo metodológico son los alumnos los que trabajan solos o de forma cooperativa para buscar posibles respuestas a las preguntas planteadas.

AC: Actividades de contraste. Es la fase principal del CIMA, los alumnos exponen sus primeros resultados y de forma colectiva y cooperativa, a través de diversas actividades de contraste, se avanza para resolver las dificultades que van surgiendo entre todos.

SINT: Sintesis. Antes de pasar a la siguiente pregunta, y después de que los alumnos sigan avanzando en la resolución de las cuestiones planteadas, se recogen en síntesis los principales aspectos puestos de manifiesto. 
Secuencia de actividades coherente con dicho modelo partiendo de los problemas señalados.

Tabla 1: Sesión primera CIMA

SESIÓN 1: En esta sesión hacemos una breve presentación de la asignatura y del CIMA. Y trabajamos la pregunta 1 del CIMA: ¿qué es la renta en el IRPF? (2 horas y media)

1a FASE: Presentación de la asignatura y el contenido del CIMA (20 minutos) (PR)

\begin{tabular}{|l|l|l|}
\hline Tipo de actividad & Presentación & Tiempo \\
\hline \multirow{5}{*}{ Actividad 1 (PR) } & $\begin{array}{l}\text { Breve presentación de la asignatura: } \\
\text { programa y sistema de evaluación } \\
\text { y criterios de calificación de la } \\
\text { asignatura. }\end{array}$ & 10 minutos \\
\hline \multirow{5}{*}{ Actividad 2 (PR) } & $\begin{array}{l}\text { Presentación de la pregunta global } \\
\text { que orientará nuestro CIMA: ¿Cuáles } \\
\text { son los aspectos fundamentales de } \\
\text { una renta gravada por el IRPF? Dicha } \\
\text { pregunta a su vez se descompone en }\end{array}$ & 10 minutos \\
& $\begin{array}{l}\text { tres preguntas parciales: 1) ¿qué es la } \\
\text { renta en el IRPF? 2) ¿quiénes son los } \\
\text { contribuyentes? 3) ¿cuándo se exige el }\end{array}$ & \\
\hline IRPF? & \\
\hline
\end{tabular}

2a FASE: comenzamos a trabajar la pregunta 1 del CIMA: ¿qué es la renta en el IRPF? (20 minutos) (IA)

\begin{tabular}{|l|l|l|}
\hline & $\begin{array}{l}\text { Se trabaja la primera pregunta del } \\
\text { cuestionario, y se pide a los alumnos } \\
\text { que comenten qué recursos utilizan } \\
\text { habitualmente. Se recogen en un } \\
\text { listado cuáles las fuentes que les van } \\
\text { a permitir acceder a recursos que les } \\
\text { sirvan en el Máster y en su futura vida } \\
\text { profesional. Consideramos importante } \\
\text { que hagan referencia a la web de la } \\
\text { AEAT. }\end{array}$ & 20 minutos \\
\hline $\begin{array}{l}\text { 3a FASE: Continuamos trabajando la pregunta 1 del CIMA: ¿qué es la renta } \\
\text { en el IRPF? (90 minutos) (AC) }\end{array}$ \\
\hline
\end{tabular}

Jornadas de Formación e Innovación Docente del Profesorado | № 2 (2019) Esta obra se distribuye con la licencia Creative Commons Reconocimiento-NoComercial-SinObraDerivada Internacional (CC BY-NC-ND 4.0.) 
Actividad 4 (AC1)

Buscaremos la LIRPF y su Reglamento en el buscador de la web de la AEAT que remite al del Boletín Oficial del Estado. Planteamos la pregunta: ¿qué es la renta en el IRPF? Se abre un debate en clase y se recogen en la pizarra las distintas rentas que deben ir comentando sus compañeros. Así analizamos el artículo 6 de la Ley del IRPF. Se les proporciona un listado extenso de diversas manifestaciones de rentas que pudieran estar sujetas a este impuesto (supuesto práctico núm. 1), y se hacen grupos de tres alumnos que tratarán de identificar cada uno de esos ejemplos con algunos de los componentes de la renta gravada en el IRPF (así se dará respuesta a la subpregunta: ¿cuáles son los componentes de la renta gravada?).

Esta actividad es continuación de la anterior, se realiza un debate de la clase en el que con un moderador (preferiblemente un alumno) se les va a pedir a cada grupo que, a través de su portavoz, exponga la clasificación de los distintos ejemplos que ha trabajado y que señale en qué se basa para llegar a esa conclusión. Entre todos los grupos se resolverá el supuesto práctico aclarando dudas y conceptos fundamentales. Con esta actividad se resuelve esa primera subpregunta.

30 minutos

30 minutos

Actividad 5 (AC2)

30

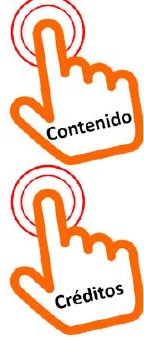

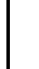


Actividad 6 (AC3)

Nueva actividad de contraste consistente en la resolución de un nuevo supuesto práctico para dar respuesta a otra de las subpreguntas: ¿puede haber alguna renta exenta? Se vuelve a trabajar en clase de forma colectiva, y entre todos -siguiendo un orden- se lee la larga lista de supuestos en el artículo 7 LIRPF y 30 minutos se comentan las dificultades que vayan apareciendo. Se les entrega el supuesto práctico núm. 2 sobre supuestos de rentas exentas del citado artículo 7 para que vean con claridad cómo se producen estas situaciones en casos reales.

4aa FASE: Síntesis. (20 minutos) (SINT)

Se concluye resaltando las ideas principales que nos han permitido formular las soluciones a estas dos preguntas que sirven para resolver

Actividad 7 (SINT) el "aspecto material" del Hecho imponible del IRPF.

Tabla 2: Sesión segunda CIMA

SESIÓN 2: En esta sesión trabajaremos la pregunta 2 del CIMA: ¿quiénes son contribuyentes en el IRPF? ( 2 horas y media)

1a FASE: Presentación (15 minutos) (PR)

\begin{tabular}{|l|l|l|}
\hline Tipo de actividad & Presentación & Tiempo \\
\hline Actividad 1 (PR) (IA) & $\begin{array}{l}\text { Se plantea la segunda pregunta de } \\
\text { CIMA: ¿quiénes son contribuyentes }\end{array}$ & \\
& $\begin{array}{l}\text { en el IRPF? Se les pide que } \\
\text { comenten quiénes creen ellos que } \\
\\
\text { son los contribuyentes en el IRPF. }\end{array}$ & \\
\hline
\end{tabular}

2a FASE: Comenzamos a trabajar la pregunta la pregunta 2 del CIMA: ¿Quiénes son contribuyentes en el IRPF? (20 minutos) (IA)

Jornadas de Formación e Innovación Docente del Profesorado | № 2 (2019) Esta obra se distribuye con la licencia Creative Commons Reconocimiento-NoComercial-SinObraDerivada Internacional (CC BY-NC-ND 4.0.) 


\begin{tabular}{|c|c|c|}
\hline Actividad 2 (IA) & $\begin{array}{l}\text { Trabajamos en grupos pequeños } \\
\text { de } 3 \text { alumnos, se les pide } \\
\text { que piensen en ejemplos de } \\
\text { contribuyentes que ellos conozcan, } \\
\text { y que intercambien sus opiniones. } \\
\text { Fomentamos así que no sólo } \\
\text { hablen de aquellos que cumplen } \\
\text { sus obligaciones tributarias, } \\
\text { sino que aparezcan casos de } \\
\text { contribuyentes que incumplen las } \\
\text { mismas, para tratar la cuestión de } \\
\text { fraude fiscal. Se abre un pequeño } \\
\text { debate estableciendo un orden de } \\
\text { intervenciones, y se recogen en la } \\
\text { pizarra los principales ejemplos } \\
\text { que los alumnos han comentado. }\end{array}$ & 20 minutos \\
\hline Actividad 3 (AC1) & $\begin{array}{l}\text { Actividad de contraste que } \\
\text { consiste en que los alumnos } \\
\text { traten de encajar los supuestos } \\
\text { de contribuyentes que hemos } \\
\text { comentado en los artículos } 8 / 10 \\
\text { LIRPF. Además, se les pide que } \\
\text { piensen en alguna noticia que } \\
\text { fundamente algún caso que } \\
\text { pueda ser reflejo de lo que alli } \\
\text { queda expuesto. Es muy probable } \\
\text { que aparezcan los casos recientes } \\
\text { de personas famosas que se van } \\
\text { de nuestro país a territorios de } \\
\text { baja tributación para no pagar el } \\
\text { IRPF, que queremos trabajar en la } \\
\text { siguiente actividad. }\end{array}$ & 20 minutos \\
\hline Actividad 4 (AC2) & $\begin{array}{l}\text { Para abordar las cuestiones de } \\
\text { los cambios de residencia vemos: } \\
\text { https://www.youtube.com / } \\
\text { watch?v=zAGjaRpbWcg video que } \\
\text { refleja situaciones de este tipo } \\
\text { relacionadas con los Youtubers } \\
\text { y deportistas conocidos. Antes } \\
\text { de ver el video les diré a los } \\
\text { alumnos que tomen nota de todas } \\
\text { las cuestiones que les parezcan } \\
\text { relevantes respecto a los artículos } \\
\text { 8/10 LIRPF para que estén atentos } \\
\text { durante la proyección. }\end{array}$ & 10 minutos \\
\hline
\end{tabular}




\begin{tabular}{|c|c|c|}
\hline Actividad 5 (AC3) & $\begin{array}{l}\text { Para dar respuesta a la } \\
\text { subpregunta planteada en el } \\
\text { mapa de contenidos: ¿todos los } \\
\text { contribuyentes son residentes?, } \\
\text { debatimos sobre el video. Les } \\
\text { dejaremos que primero en grupos } \\
\text { detres durante } 10 \text { minutosaborden } \\
\text { las cuestiones fundamentales } \\
\text { que crean que se han puesto } \\
\text { de manifiesto en el video, para } \\
\text { posteriormente afrontar el } \\
\text { debate, de la misma forma que } \\
\text { lo expuesto anteriormente, por } \\
\text { lo que no reproduzco los mismos } \\
\text { comentarios. }\end{array}$ & 35 minutos \\
\hline Actividad 6 (AC4) & $\begin{array}{l}\text { Para dar respuesta a otra de las } \\
\text { subpreguntas formuladas: ¿a } \\
\text { quién corresponden las rentas?, } \\
\text { utilizaremos la LIRPF y a través de } \\
\text { un nuevo supuesto práctico núm. } \\
3 \text { trabajaremos los criterios de } \\
\text { individualización de la renta del } \\
\text { artículo } 11 \text { de la LIRPF. En este caso } \\
\text { el trabajo se va a desarrollar de } \\
\text { forma personal durante } 15 \text { minutos } \\
\text { y en los restantes } 10 \text { minutos se } \\
\text { resuelven dichas preguntas y se } \\
\text { aclaran las dudas. Tomaré nota } \\
\text { de las intervenciones de cara a la } \\
\text { calificación final. }\end{array}$ & 35 minutos \\
\hline \multicolumn{3}{|c|}{ 4aㅡ FASE: Sintesis. (15 minutos) (SINT) } \\
\hline Actividad 7 (SINT) & $\begin{array}{l}\text { Se concluye resaltando las ideas } \\
\text { principales que nos han permitido } \\
\text { formular las soluciones a estas } \\
\text { dos preguntas que sirven para } \\
\text { resolver el "aspecto personal" del } \\
\text { hecho imponible del IRPF. }\end{array}$ & 15 minutos \\
\hline
\end{tabular}

Tabla 3: Sesión tercera del CIMA

\section{SESIÓN 3: En esta sesión trabajaremos la pregunta 3 del CIMA: ¿cuándo se exige el IRPF? (3 horas)}


1a FASE: Presentación (10 minutos) (PR)

\begin{tabular}{|l|l|l|}
\hline $\begin{array}{l}\text { Tipo de } \\
\text { actividad }\end{array}$ & Presentación & Tiempo \\
\hline Actividad 1 (PR) & $\begin{array}{l}\text { Se plantea la tercera pregunta parcial: } \\
\text { ¿cuándo se exige el IRPF? Con ella } \\
\text { abordaremos el aspecto temporal del } \\
\text { Hecho imponible del impuesto: devengo } \\
\text { y exigibilidad. }\end{array}$ & \\
\hline
\end{tabular}

2a FASE: Comenzamos a trabajar la pregunta la pregunta 3 del CIMA: ¿Quiénes son contribuyentes en el IRPF? (20 minutos) (IA)

\begin{tabular}{|c|c|c|}
\hline Actividad 2 (IA) & $\begin{array}{l}\text { Trabajo en grupos pequeños de } 3 \\
\text { alumnos. Se les pegunta qué conocen } \\
\text { en relación con la exigibilidad del IRPF: } \\
\text { nacimiento de la obligación de pagar, } \\
\text { devengo, plazos de pago...al respecto, } \\
\text { para que con sus intervenciones } \\
\text { pongan de manifiesto los principales } \\
\text { problemas que se plantean y que serán } \\
\text { los que trabajemos en las actividades } \\
\text { posteriores. }\end{array}$ & 20 minutos \\
\hline \multicolumn{3}{|c|}{$\begin{array}{l}\text { 3a FASE: Continuamos trabajando la pregunta } 3 \text { del CIMA ( } 90 \text { minutos) } \\
\text { (AC) }\end{array}$} \\
\hline Actividad 3 (AC1) & $\begin{array}{l}\text { Leemos los artículos } 12 \text { y } 13 \text { de la LIRPF } \\
\text { que no plantean grandes dificultades, } \\
\text { y fundamentalmente el artículo } 14 \text { que } \\
\text { es bastante más complejo. Utilizando } \\
\text { los mismos grupos formados en la fase } \\
\text { anterior, se les pide a los alumnos que } \\
\text { busquen ejemplos de contribuyentes } \\
\text { que encajen en esos supuestos que } \\
\text { se recogen en el citado artículo } 14 \\
\text { LIRPF. Para guiarles en el estudio se les } \\
\text { proporciona un nuevo supuesto práctico } \\
\text { que deben intentar resolver en ese } \\
\text { tiempo con ayuda de las herramientas } \\
\text { que vienen siendo utilizadas en las } \\
\text { actividades que venimos realizando en } \\
\text { clase. }\end{array}$ & 45 minutos \\
\hline
\end{tabular}

Jornadas de Formación e Innovación Docente del Profesorado | № 2 (2019) Esta obra se distribuye con la licencia Creative Commons Reconocimiento-NoComercial-SinObraDerivada Internacional (CC BY-NC-ND 4.0.) 
Para terminar esta tercera fase, trataremos de dar respuesta a la última de las subpreguntas planteadas: ¿cómo se imputan las rentas temporalmente? Pediremos a los portavoces de los distintos grupos que analicen uno de los supuestos que hayan trabajado en la actividad anterior, y entre todos resolveremos el supuesto práctico, solucionando las dudas que hayan podido surgir utilizando la fórmula del debate que ellos ya conocen.

4aㅡ FASE: Síntesis. (60 minutos) (SINT)

Actividad 5 (SINT)
En esta fase vamos a sintetizar las ideas extraídas de la actividad anterior para dar respuesta a la tercera pregunta parcial formulada sobre cuándo se exige la renta y recogeremos todas las conclusiones de las fases anteriores poniéndolas en relación, pues todas ellas resuelven la pregunta global que planteamos inicialmente sobre los aspectos fundamentales de la renta gravada en el IRPF. Por eso su duración es mayor que en las otras fases y el modelo metodológico se somete a un pequeño cambio. Para que los alumnos sean conscientes de la importancia de los conceptos trabajados utilizaremos el modelo D-100 IRPF, y nos detendremos en las casillas que reflejan los aspectos estudiados en estas sesiones del CIMA.

\section{Cuestionario de diagnóstico inicial-final.}

Este es el cuestionario que planteo como punto de partida. Voy a aplicarlo de forma anónima, y puesto que el CIMA se va a desarrollar en un Máster, en el que la procedencia y la formación de los alumnos no es uniforme, considero que es una buen sistema para poder conocer cuál es el nivel del que se parte, para adaptar el contenido de 
las actividades que se van a programar, y en su caso, poder utilizar algunos recursos que permitan que todos los alumnos consigan alcanzar el nivel que se espera de unos estudios de especialización de este tipo.

Estas son las preguntas del cuestionario:

1) Imagínate que eres un afamado asesor fiscal y que tienes una cartera de clientes importante, ya que estás especializado en el IRPF. Como es lógico tendrás que hacer frente a problemas que resultan especialmente complejos, ¿dónde buscarías información fiable sobre las cuestiones que te plantearan tus clientes?

2) Dado que conoces en profundidad el IRPF, te proponen participar en una Comisión de expertos para redactar una modificación del texto legal en relación con los distintos componentes de la renta. ¿Podrías indicar cuáles son los componentes de la renta regulados por la LIRPF? ¿Piensas que habría que prescindir de alguno de ellos o incluir algún otro? ¿Por qué?

3) En esa reforma proyectada, y puesto que se necesitan más recursos que hagan frente a la "desaceleración económica" que se prevé en los próximos ejercicios, se modificará la tributación de algunos tipos de renta. ¿Consideras que cualquier manifestación de renta debe quedar sometida al IRPF? Justifica la respuesta a esta pregunta tanto si es afirmativa, como si por el contrario piensas que en algunos casos esto no debiera ser así.

4) Otro de los aspectos problemáticos en esa reforma que se pretende llevar a cabo es la determinación de a quién/quienes les "corresponden" esas rentas, ya que con la globalización económica todo resulta más complejo: ¿cuál es tu opinión al respecto? 
5) Por último, se pretenden analizar problemas en relación con el momento en que las rentas deben quedar sometidas al IRPF, te piden que comentes si has tenido recientemente algún problema relacionado con esta cuestión.

\section{Aplicación de CIMA}

\section{Relato resumido de las sesiones}

Considero que en general la experiencia ha sido positiva y que se lograron alcanzar los objetivos inicialmente propuestos. Los alumnos mostraron un gran interés desde el principio, asumiendo las tareas que se les planteaba fundamentalmente a través de trabajos en grupo y posteriormente debates sobre las cuestiones que se habian trabajado previamente en dichos grupos. Aunque inicialmente costó trabajo que la participación se generalizara, poco a poco se fueron logrando avances hasta el punto de necesitar más tiempo del programado para la realización de los debates debido al grado de participación de los alumnos en las clases. Me resultó llamativo que, pese a que las sesiones eran muy largas, los alumnos no pedían realizar el descanso intermedio, pues considero que las clases fueron especialmente dinámicas.

El peso fundamental de las actividades de contraste fue llevado por los alumnos que fueron capaces de asumir un rol activo de forma natural, permitiendo que mi papel pasara a un segundo plano y se limitara a resolver las dudas principales y a guiarles hacia nuestro objetivo a través de unas actividades programadas que permitieron ir resolviendo las distintas preguntas y el problema planteado en el CIMA. Considero que la madurez del grupo ha sido un acierto de cara al desarrollo de este ciclo de mejora. 
El mapa de contenidos y el cuestionario inicial me sirvieron como hilo conductor en todo momento, y aunque tuve que adaptarme en algunas ocasiones a los tiempos que marcaban las clases, me ayudó a no perderme y alcanzar el objetivo inicial. Por otra parte, se trabajaron no solo aspectos puramente teóricos sino otros de contenido actitudinal que considero que en una disciplina como la nuestra son especialmente relevantes, me refiero a aquellos especialmente relacionados con la lucha contra el fraude fiscal.

\section{Evolución del aprendizaje de los alumnos.}

Antes de iniciar el ciclo de mejora docente y después de finalizar el mismo, se pidió a los alumnos del curso que cumplimentaran el cuestionario indicado anteriormente, para mantener el anonimato se les pidió que reflejaran el nombre del Instituto o colegio en el que cursaron $\mathrm{Ba}$ chillerato. Dado que el cuestionario inicial lo cumplimentaron solo 24 alumnos, ha sido con esos alumnos con los que hemos trabajado para la elaboración de las escaleras de aprendizaje.

Aunque, como hemos dicho anteriormente, el cuestionario inicial tenía 5 preguntas, aquí recogemos sólo dos de ellas. En todas se observa un avance en el aprendizaje, a mi juicio, nos solo motivado por el CIMA, sino por el interés del alumnado de Máster que ve que se aproxima su salida al mundo profesional. Dichas escaleras se corresponden con las figuras no 3 y 4 que se incluyen a continuación. En ellas, los porcentajes de color rojo corresponden a las respuestas iniciales al cuestionario y los de color azul a las respuestas finales al mismo.

Jornadas de Formación e Innovación Docente del Profesorado I № 2 (2019) Esta obra se distribuye con la licencia Creative Commons Reconocimiento-NoComercial-SinObraDerivada 


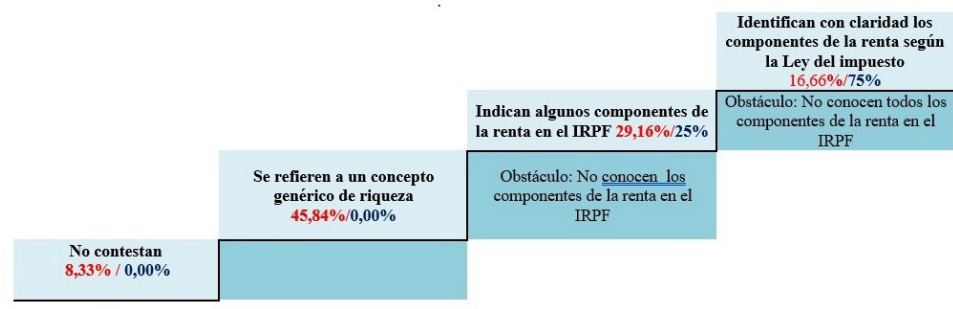

Figura 3. Escalera de aprendizaje pregunta 3.

PREGUNTA 3: ¿cualquier manifestación de renta debería quedar gravada en el IRPF?

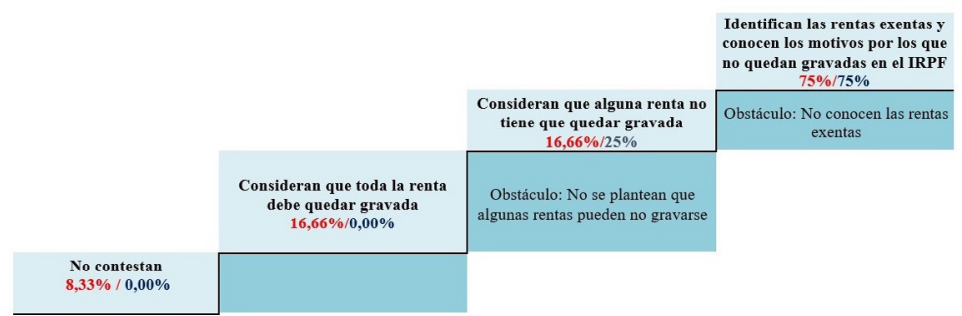

Figura 4. Escalera de aprendizaje pregunta 4.

Una vez planteadas las preguntas hemos llevado a cabo un análisis de la evolución de los alumnos que contestaron el cuestionario inicialmente. Como hemos indicado el resultado ha sido claramente satisfactorio, pues todos los alumnos han avanzado en su aprendizaje y han superado los obstáculos que les impedian pasar al siguiente escalón, alcanzando la mayoría el nivel más alto que se esperaba de ellos.

En el siguiente cuadro pueden verse los resultados alcanzados, indicando en cada uno de ellos si ha mejorado o se han mantenido en el mismo nivel. 
Tabla 4. Tabla de evaluación de los modelos iniciales y finales de los estudiantes.

EVALUACIÓN INDIVIDUAL DE LOS MODELOS INICIALES Y FINALES DE LOS ESTUDIANTES

$\uparrow$ Mejora $\rightarrow$ Se mantiene

\begin{tabular}{|c|c|c|c|c|c|c|}
\hline \multirow{2}{*}{ ALUMNO } & \multicolumn{3}{|c|}{$\begin{array}{c}\text { CUESTIONARIO } \\
\text { INICIAL }\end{array}$} & \multicolumn{3}{|c|}{$\begin{array}{c}\text { CUESTIONARIO } \\
\text { FINAL }\end{array}$} \\
\hline & P1 & P2 & P3 & P1 & P2 & P3 \\
\hline 1 & $\mathrm{C}$ & $\mathrm{C}$ & B & $\mathrm{A} \uparrow$ & $\mathrm{B} \uparrow$ & $\mathrm{A} \uparrow$ \\
\hline 2 & $\mathrm{~A}$ & A & $\mathrm{A}$ & $\mathrm{A} \rightarrow$ & $\mathrm{A} \rightarrow$ & $\mathrm{A} \rightarrow$ \\
\hline 3 & $\mathrm{D}$ & $\mathrm{C}$ & D & $\mathrm{B} \uparrow$ & $\mathrm{A} \uparrow$ & $\mathrm{B} \uparrow$ \\
\hline 4 & B & $\mathrm{C}$ & B & $\mathrm{A} \uparrow$ & $\mathrm{A} \uparrow$ & $\mathrm{A} \uparrow$ \\
\hline 5 & D & C & B & $\mathrm{A} \uparrow$ & $\mathrm{A} \uparrow$ & $\mathrm{A} \uparrow$ \\
\hline 6 & $\mathrm{~A}$ & $\mathrm{C}$ & $\mathrm{A}$ & $\mathrm{A} \rightarrow$ & $\mathrm{A} \uparrow$ & $\mathrm{A} \rightarrow$ \\
\hline 7 & $\mathrm{~A}$ & $\mathrm{~A}$ & $\mathrm{~A}$ & $\mathrm{~A} \rightarrow$ & $\mathrm{A} \rightarrow$ & $\mathrm{A} \rightarrow$ \\
\hline 8 & B & $\mathrm{D}$ & $\mathrm{C}$ & $\mathrm{A} \uparrow$ & $\mathrm{A} \uparrow$ & $\mathrm{B} \uparrow$ \\
\hline 9 & $\mathrm{~A}$ & $\mathrm{~A}$ & B & $\mathrm{A} \rightarrow$ & $\mathrm{A} \rightarrow$ & B \\
\hline 10 & $\mathrm{C}$ & $\mathrm{C}$ & B & $\mathrm{A} \uparrow$ & $\mathrm{B} \uparrow$ & $\mathrm{A} \uparrow$ \\
\hline 11 & B & B & B & $\mathrm{A} \uparrow$ & $\mathrm{A} \uparrow$ & $\mathrm{A} \uparrow$ \\
\hline 12 & $\mathrm{D}$ & $\mathrm{D}$ & $\mathrm{D}$ & $\mathrm{A} \uparrow$ & $\mathrm{B} \uparrow$ & $\mathrm{B} \uparrow$ \\
\hline 13 & $\mathrm{D}$ & $\mathrm{C}$ & $\mathrm{C}$ & $\mathrm{B} \uparrow$ & $\mathrm{B} \uparrow$ & $\mathrm{A} \uparrow$ \\
\hline 14 & $\mathrm{~A}$ & $\mathrm{C}$ & B & $\mathrm{A} \rightarrow$ & $\mathrm{A} \uparrow$ & $\mathrm{A} \uparrow$ \\
\hline 15 & B & B & B & $\mathrm{A} \uparrow$ & $\mathrm{A} \uparrow$ & $\mathrm{A} \uparrow$ \\
\hline 16 & $\mathrm{D}$ & $\mathrm{C}$ & $\mathrm{C}$ & $\mathrm{B} \uparrow$ & $\mathrm{A} \uparrow$ & $\mathrm{A} \uparrow$ \\
\hline 17 & $\mathrm{C}$ & B & B & $\mathrm{A} \uparrow$ & $\mathrm{A} \uparrow$ & $\mathrm{A} \uparrow$ \\
\hline 18 & $\mathrm{D}$ & $\mathrm{C}$ & $\mathrm{C}$ & $\mathrm{B} \uparrow$ & $\mathrm{B} \uparrow$ & $\mathrm{B} \uparrow$ \\
\hline 19 & $\mathrm{C}$ & $\mathrm{C}$ & B & $\mathrm{B} \uparrow$ & $\mathrm{B} \uparrow$ & $\mathrm{B} \rightarrow$ \\
\hline 20 & $\mathrm{~B}$ & $\mathrm{~B}$ & $\mathrm{~A}$ & $\mathrm{~A} \uparrow$ & $\mathrm{A} \uparrow$ & $\mathrm{A} \rightarrow$ \\
\hline 21 & B & B & B & $\mathrm{A} \uparrow$ & $\mathrm{A} \uparrow$ & $\mathrm{A} \uparrow$ \\
\hline 22 & $\mathrm{~B}$ & $\mathrm{~B}$ & $\mathrm{~B}$ & $\mathrm{~A} \uparrow$ & $\mathrm{A} \uparrow$ & $\mathrm{A} \uparrow$ \\
\hline 23 & B & B & B & $\mathrm{A} \uparrow$ & $\mathrm{A} \uparrow$ & $\mathrm{A} \uparrow$ \\
\hline 24 & $\mathrm{~A}$ & $\mathrm{~A}$ & $\mathrm{~A}$ & $\mathrm{~A} \rightarrow$ & $\mathrm{A} \rightarrow$ & $\mathrm{A} \rightarrow$ \\
\hline
\end{tabular}

\section{Evaluación del CIMA.}

\section{Cuestiones a mantener y cambios a introducir para un futuro Ciclo de Mejora más amplio}

- Cuestiones a mantener:

a) Reconfigurar el aula: quitar al profesor del centro de atención del alumno y que ésta se centre en la materia sobre la que se esté trabajando. Poner a los estudiantes a trabajar y confiar en su capacidad. Asumir como docentes un papel de "guía"

Jornadas de Formación e Innovación Docente del Profesorado | № 2 (2019) Esta obra se distribuye con la licencia Creative Commons Reconocimiento-NoComercial-SinObraDerivada 
en la preparación del contenido de las actividades y de "actores secundarios" en su ejecución.

b) Definir los distintos tipos de contenidos conceptuales, procedimentales y actitudinales. No todos los contenidos tienen la misma importancia, en este sentido el mapa de contenidos me ha ayudado a clarificar la posición de las distintas materias relacionadas con el problema fundamental determinando su posición en el mismo, centrando el objetivo perseguido con el desarrollo del CIMA.

c) Trabajar los contenidos en torno a un problema central y clave, como forma de analizar los contenidos de una asignatura a través de una secuencia de actividades bien programada y detallada.

d) Conocer y analizar los modelos mentales de los estudiantes mediante cuestionarios y escaleras de aprendizaje que nos ayuden a definir los obstáculos y los niveles de nuestros alumnos.

- Cambios a introducir en un futuro

a) Introducir la evaluación del estudiante durante el proceso de enseñanza aprendizaje utilizando el portafolio, para que sean conscientes de la importancia del papel que asumen.

b) Introducir la evaluación del docente y del diseño didáctico durante el proceso a través del diario del profesor y encuestas de opinión de los estudiantes.

c) Seguir trabajando con la selección y definición de los principales contenidos que se pretenden desarrollar a través de futuros problemas. 
Considero que voy a implantar los aspectos que he señalado en el apartado anterior, pues la experiencia desarrollada en este CIMA me ha permitido constatar que los alumnos tienen un interés mayor o igual que el nuestro en asumir un "rol activo" en este modelo de aprendizaje, por lo que volver al modelo tradicional sería un paso atrás que no me gustaría dar.

\section{Principios didácticos que han guiado la experiencia y que deben permanecer en el futuro}

Después del desarrollo de este curso, de la experiencia del CIMA y de la lectura de los libros que se han recomendado, considero que son estos los principios didácticos en los que me he basado:

a) Reconfiguración del aula: inversión de los papeles del alumno/profesor.

b) Elaboración de mapas de contenidos y problemas de trabajo para definir lo importante y lo secundario.

c) Diseño y aplicación de secuencias de actividades detalladas que permiten avanzar en el aprendizaje. Evaluación y desarrollo de las que mejor se adaptan a nuestros contenidos -en nuestro caso tienen un papel fundamental las actividades de contraste-, y cambio de las que no nos sirven.

d) Trabajo con los modelos mentales de los estudiantes, a través de cuestionarios útiles para el proceso de evaluación del aprendizaje, nos permite detectar obstáculos que permitan hacer modificaciones tanto en contenidos como en secuencias de actividades. 
e) Implementación de técnicas de autoevaluación y de evaluación de las actividades de los alumnos a través del diario de clase, encuestas y portafolio. 


\section{Referencias Bibliográficas}

BAIN, K. (2005) Lo que hacen los mejores profesores universitarios, Valencia, Publicaciones de la Universidad de Valencia.

FINKEL, D. (2008). Dar clase con la boca cerrada. Valencia: Publicaciones Universidad de Valencia.

PORLÁN, R. (2017). Enseñanza universitaria. Cómo mejorarla. Madrid: Ediciones Morata, S.L 